\title{
Dritter Nachtrag zur Rüsselkäfer-Fauna der Schweiz (Coleoptera, Curculionoidea)
}

Third addition to the checklist of Swiss weevils (Coleoptera, Curculionoidea)

\section{Christoph Germann ${ }^{1}$}

1 Kurator Biowissenschaften, Naturhistorisches Museum Basel, Augustinergasse 2, CH-4001 Basel

http://zoobank.org/B9075394-5E9E-4C70-9F66-79B0A8572758

Corresponding author: Christoph Germann (Christoph.Germann@bs.ch)

Received 28 June 2019

Accepted 23 October 2019

Published 6 November 2019

Academic editor:

Yannick Chittaro

\section{Key Words}

Curculionoidea

checklist

new records

resurrected name

Switzerland

\section{Zusammenfassung}

Die Anzahl verlässlich gemeldeter Taxa (Arten und Unterarten) der Curculionoidea aus der Schweiz beträgt momentan 1081. Funde von Orchestes calceatus (Germar, 1821) werden hier erstmals bestätigt. Zusätzliche Verbreitungsdaten von zehn Arten werden gegeben. Der auf subalpine und alpine Lebensräume beschränkte Phyllobius alpinus Stierlin, 1859 ist eine valide Art stat. rev., und kein Synonym zu Ph. xanthocnemus Kiesenwetter, 1852 und wird daher aus der Synonymie herausgenommen. Die Arten werden verglichen und typische Unterscheidungsmerkmale werden aufgeführt.

\begin{abstract}
The number of reliably recorded taxa (species and subspecies) of Curculionoidea from Switzerland counts at present 1081. Records of Orchestes calceatus (Germar, 1821) are confirmed here for the first time. Additional data on distribution is given for ten species. Phyllobius alpinus Stierlin, 1859, restricted to subalpine and alpine habitats, is a valid species stat. rev., and not a synonym of $P h$. xanthocnemus Kiesenwetter, 1852 and thus resurrected from synonymy. Both species are compared and characters for differentiation are provided.
\end{abstract}

\section{Einleitung}

Die im 2010 vorgestellte Checkliste der schweizerischen Rüsselkäfer (Germann 2010) und deren Supplement im Folgejahr (Germann 2011), wurden durch zwei Nachträge ergänzt (Germann 2013, 2017). Seither erfolgten weitere Entdeckungen. Das Vorkommen von Polydrusus sparsus Gyllenhal, 1834 konnte erfreulicherweise durch einen aktuellen Nachweis bestätigt werden (Germann and Chittaro 2019). Germann and Graf (2018) meldeten Mecinus pascuorum (Gyllenhal, 1813) erstmals aus der Zentralschweiz und Borer et al. (2018) berichteten über Käfer aus den Merian-Gärten in Basel, darunter Nachweise von Eusomus ovulum Germar, 1824, einer in der Schweiz wenig gefundenen, sehr lokal vorkommenden und wenig mobilen Art xerothermer Lebensräume. Während zweier Biodiversitäts-Veranstaltungen im 2018 in der Zentralschweiz, im Gebiet von Fryberg Kärpf (GL) und auf dem Urnerboden (UR) wurden zudem grössere Aufsammlungen unternommen, und im Val Müstair wurden während eines GEO-Tages der Artenvielfalt im Juni 2019 weitere Funde zusammengetragen. Die Resultate - darunter einige Erstnachweise - sollen auch innerhalb nützlicher Frist publiziert werden. Um den erneuten Anpassungen in Nomenklatur, Taxonomie und Faunistik gerecht zu werden, wird hier ein dritter Nachtrag zur Checkliste gegeben. Zudem werden Funddaten von wenig gemeldeten und bemerkenswerten Arten aufgeführt und der Status einer Art wird revidiert. 


\section{Material und Methoden}

Folgende Abkürzungen werden verwendet:

cCG Sammlung Christoph Germann (Rubigen)

cGF Sammlung Georg Frey (im NMB)

cHB Sammlung Hermann Blöchlinger (Erschmatt)

cSB Sammlung Stève Breitenmoser (Changins)

ETHZ Eidgenössisch-Technische Hochschule Zürich

HJB Sammlung Hansjörg Brägger (Amriswil)

NMB Naturhistorisches Museum Basel

NMSO Naturmuseum Solothurn

Es werden Schweizer Koordinaten angegeben und zusätzlich die internationalen Koordinaten (lat./long.) in eckigen Klammern.

Folgende Vergleichsexemplare wurden zwecks Illustration des revidierten Status von Phyllobius alpinus fotografiert: Ph. alpinus $\widehat{\overbrace{}}$ Italy, Piemonte, Valle Formazza, Vannino, ob. Valdo, Sagersboden, 674790N/136683E, [46²2'38"N, $\left.8^{\circ} 24^{\prime} 38^{\prime \prime E}\right] 1770$ m, 4.7.2010, leg. C. Germann; 9 Austria, Steiermark, Rottenmanner Tauern, Hengst, 4706'36"N, 1309'07"E, 1500 m, 29.7.2007, leg. C. Germann (cCG). Ph. xanthocnemus $\widehat{\jmath}$ Canterets, Hautes Pyrenées, coll. J. Breit (cGF); ㅇ France, Hautes Pyrenées, Gavarnie, E-side, $42^{\circ} 43^{\prime} 20^{\prime \prime} \mathrm{N}, 0^{\circ} 00^{\prime} 01^{\prime \prime W}, 1650 \mathrm{~m}, 9.8 .2007$, leg. C. Germann (cCG). Die Nomenklatur folgt dem neusten Katalog von Alonso-Zarazaga et al. (2017).

\section{Resultate}

\section{Neue Funddaten \\ Ceutorhynchinae}

\section{Drupenatus nasturtii (Germar, 1824)}

Untersuchte Exemplare. 3 Ex., BE, Rubigen, Hechteloch, Schwarzbach, 608245N/193083E [4653'20"N, 7³2'48"E], 520 m, 10.6.2019, leg. C. Germann (cCG).

Bemerkung. Die letzten Funde lagen aus den 70 er Jahren vor (Aarwangen BE, leg. \& coll. A. Linder ETHZ), hier erstmals aktuelle Funde der auf Feuchtgebiete beschränkten Art.

\section{Neophytobius granatus (Gyllenhal, 1836)}

Untersuchte Exemplare. 2 , BE, Spiez, Einigen, Kanderdelta, 31.5.2017, leg. A. Jaun via W. Marggi (cCG, NMSO).

Bemerkung. Bisher lagen nur wenige und überwiegend ältere Nachweise der Art vor. Erste Nachweise aus der biogeographischen Region Alpennordflanke (Germann 2010).

\section{Phrydiuchus topiarius (Germar, 1824)}

Untersuchte Exemplare. 1 , VS, Turtmanntal, Unterems, Äbnet, 620000N/126200E, [46²17'13"N, 741'53"E], 1150 m, 4.11.2012, leg. H. Blöchlinger (cHB).

Bemerkung. Selten und schwierig nachzuweisende Art, an südexponierte Trockenstandorte gebunden und meist nur unter den Blättern von Salvia pratensis L. zu finden. Die Tiere sind durch ihre Färbung bestens getarnt.

\section{Stenocarus ruficornis (Stephens, 1831)}

Untersuchte Exemplare. $1 \hat{\jmath}$, BE, Rubigen, in Garten, 10.6.2018, leg. C. Germann (cCG).

Bemerkung. Bisher keine aktuellen Funde aus dem Mittelland.

\section{Cossoninae}

\section{Cossonus cylindricus C. R. Sahlberg, 1834}

Untersuchte Exemplare. 1 Ex., VD, Trélex, La Coque, Le Molard, 6.5.2018, leg. S. Breitenmoser (cSB).

Bemerkung. Bisher nur wenige und ältere Nachweise.

\section{Cryptorhynchinae}

\section{Gasterocercus depressirostris (Fabricius, 1792)}

Untersuchte Exemplare. 1 Ex., TG, Amriswil, Oberfeld, $740050 \mathrm{~N} / 267225 \mathrm{E} \quad\left[47^{\circ} 32^{\prime} 27^{\prime \prime} \mathrm{N}, 9^{\circ} 17^{\prime} 55^{\prime \prime} \mathrm{E}\right], 460 \mathrm{~m}$, 5.6.2018, leg. H. Brägger (cHB). 2 Ex., dito 18.8.2018. 1 Ex., Bern, Elfenau, 12.6.2018, leg. TeilnehmerInnen der Uni-Käferexkursion (cCG).

Bemerkung. Wie bereits von Germann (2017) erwähnt, wurden nun seit dem Erstnachweis dieser Art im 2013 stetig weitere Exemplare gefunden, vorliegend in der Nähe altständiger Eichen am Rand einer Flussaue, beim Abkeschern der Vegetation.

\section{Curculioninae}

\section{Curculio vicetinus Cussigh, 1989}

Untersuchte Exemplare. 1 Ex., BE, Rubigen, Hechteloch, $520 \mathrm{~m}$, an Gallen von Pediaspis aceris Gmelin, 1790 (Hymenoptera: Cynipidae) auf Acer pseudoplatanus L., 10.6.2019, leg. C. Germann (cCG).

Bemerkung. Erstmals im Basler Zoo im 2005 für die Schweiz nachgewiesen (Germann 2014), hier der zweite Nachweis weit ab vom Rheintal (dort eine Häufung von Funden) oder von Italien, wo die Art aus den Monti Berici bei Vicenza beschrieben wurde.

\section{Orchestes calceatus (Germar, 1821)}

Untersuchtes Exemplar. 1 , BE, Wimmis, $613970 \mathrm{~N} / 168800 \mathrm{E},\left[46^{\circ} 40^{\prime} 13^{\prime \prime N}, 7^{\circ} 37^{\prime} 16^{\prime \prime} \mathrm{E}\right], 630 \mathrm{~m}$, 23.6.2006, Auenwald, leg. C. Germann (cCG).

Bemerkungen. Bestätigung für die Schweiz. Bereits Kraatz (1874: 280) erwähnte Funde der auf Birke (Betula pendula und $B$. pubescens) lebenden, selten gefundenen Art. So erwähnt er mehrere Exemplare aus der Schweiz: "Vom semirufus [semirufus Gyllenhal, 1827 = synonym zu calceatus] besitze ich nur ein Stück von Barmen [SG] mit fast ganz abgeriebener Behaarung, welches vollkommen mit dem grössern meiner beiden Schweizer 5-mac- 

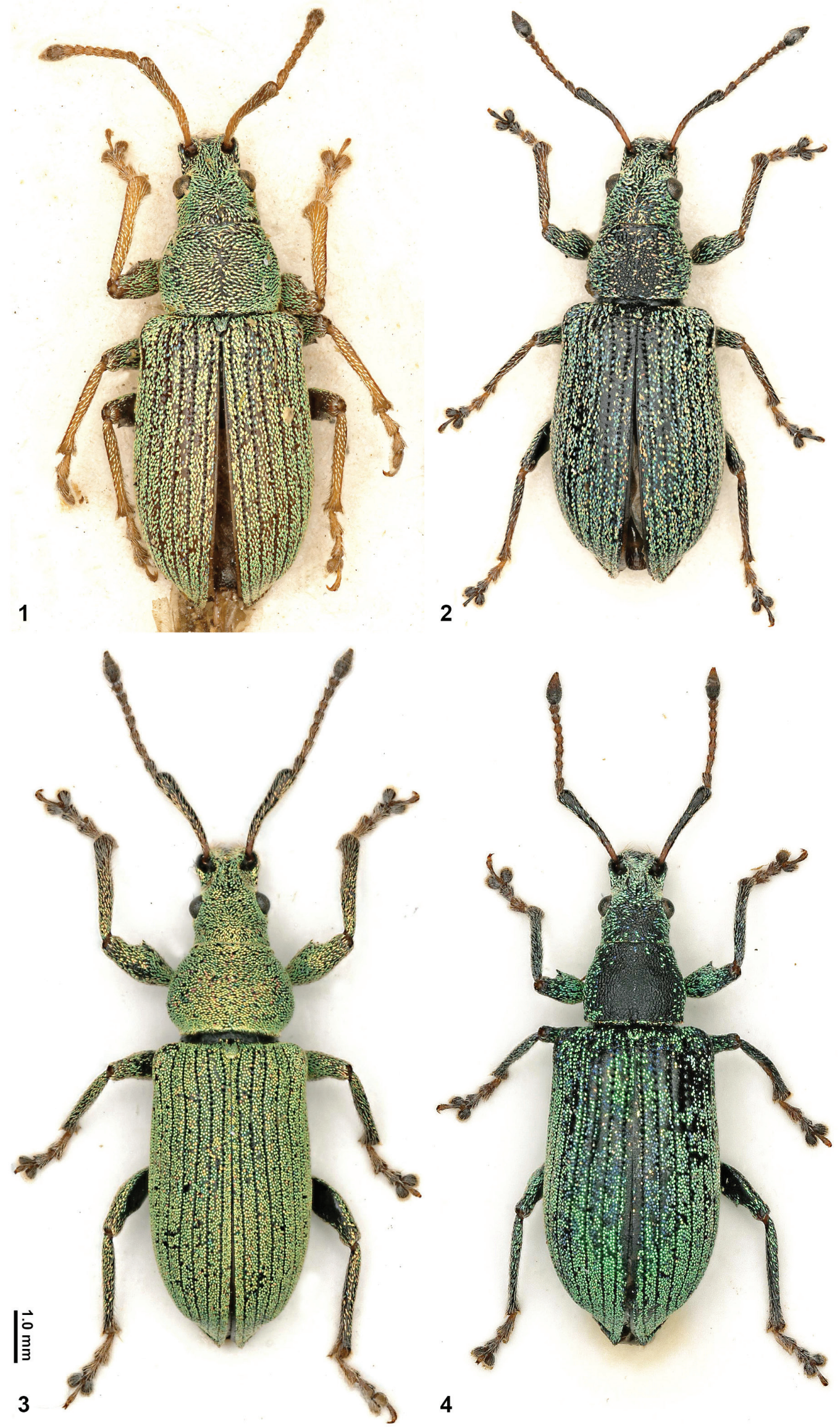

Abb. 1-4. 1-2 Männchen (Frankreich, Hautes Pyrenées, Canterets) und Weibchen (Frankreich, Hautes Pyrenées, Gavarnie) von Phyllobius xanthocnemus. 3-4 Männchen (Italia, Valle Formazza) und Weibchen (Österreich, Tauern) von Phyllobius alpinus (Fotos: C. Germann). 
ulatus [quinquemaculatus Chevrolat, 1867 = synonym zu calceatus] übereinstimmt...". Stierlin (1883) meldete O. calceatus (unter dem Synonym pubescens Gyllenhal, 1835 und als semirufus) von Bünzen und dem Bünzenmoos, später (Stierlin 1898) auch aus dem Wallis und unter quinquemaculatus zusätzlich von Aarau, Laufenburg und Schaffhausen. In der Checkliste (Germann 2010) wurde $O$. calceatus noch als Fehlmeldung eingestuft, da keine Belegtiere vorlagen, dies kann hier nun revidiert werden.

\section{Tychius cuprifer (Panzer, 1799)}

Untersuchte Exemplare. 1 Ex., VD, Prangins, Changins, $507855 \mathrm{~N} / 138920 \mathrm{E},\left[46^{\circ} 23^{\prime} 43^{\prime \prime} \mathrm{N}, 6^{\circ} 14^{\prime} 25^{\prime \prime} \mathrm{E}\right.$, $422 \mathrm{~m}$, 3-4 (Fallenfänge). 2018, leg. S. Breitenmoser (cSB). 1 Ex,. dito, 8.5.2018. 1 ex. dito, 25.5.2018.

Bemerkung. Xerothermophile Art, überwiegend an Trifolium arvense, wurde in Deutschland als verletzlich eingestuft (Rheinheimer and Hassler 2010). Auch bei uns liegen nur punktuell und vorwiegend aus Wärmegebieten Funde vor.

\section{Entiminae}

\section{Otiorhynchus indefinitus Reitter, 1912}

Untersuchte Exemplare. 10 Ex. BA, Basel-Stadt, Gundeli-Quartier, 610843N/266174E, [47³2'46"N, 7³4'57"E], bei Nacht an Hecken, 19.9.2019, leg. C. Germann (cCG, NMB).

Bemerkung. Seit 2006 (Germann 2006) sind Funde dieser früher als O. dieckmanni Magnano, 1979 bekannten Art aus der Schweiz bekannt. Nach den Erstnachweisen aus Bern hier ein weiterer individuenreicher Nachweis aus dem urbanen Basel.

\section{Hyperinae}

\section{Donus intermedius (Boheman, 1842)}

Untersuchte Exemplare. 2 Ex., BE, Vauffelin, Les Tayés, 589791N/226754E, [47¹1'30"N, 7¹8'13"E], 770 m, 2.4.2014, leg. R. Bryner (cCG).

Bemerkung. Nur wenige neue Funde von dieser allgemein selten gefundenen Art sind bekannt.

\section{Taxonomie}

Phyllobius alpinus Stierlin, 1859 bona species, status revidiert

Alonso-Zarazaga et al. (2017) führen im neusten Katalog der paläarktischen Rüsselkäfer Phyllobius alpinus Stierlin, 1859, als Synonym von Phyllobius xanthocnemus Kiesenwetter, 1852 auf. Diese Synonymie wurde von Stüben et al. (2015) vorgeschlagen und durch AlonsoZarazaga et al. (2017) schliesslich festgelegt. Nach eingehender Prüfung von Vergleichstieren konnten jedoch die bisher bekannten und auch verlässlichen Merkmale zur Unterscheidung beider Arten nach Pesarini (1981) bestätigt werden. Untersuchte Exemplare von $P h$. xanthocnemus sind durchwegs kleiner [N=6, 4.2-4.6 mm (Pesarini 1981: 4.5-5.5 mm), bei Ph. alpinus $\mathrm{N}=7,5.2-7.5 \mathrm{~mm}$ (Pesarini 1981: 4.5-7.0 mm)], vom Augenvorderrand bis Apex der Elytren dorsal gemessen), die Dornen der Femora sind schwächer ausgeprägt, der Kopf ist weniger markant eckig geformt, die Augen weniger prominent hervorstehend, die Pterygien schwächer vorgezogen, das Pronotum ist, vorallem beim Männchen, weniger ausgeprägt glockenförmig (markant glockenförmig beim Männchen von $P h$. alpinus), die Schuppen sind tropfenförmig (rund bei Ph. alpinus) und die Elytren schmaler (Abb. 1-4). Somit muss Phyllobius alpinus als valide Art gelten und die Synonymie wird hiermit rückgängig gemacht (stat. rev.). Ph. alpinus ist weit verbreitet, von den Karpathen und Sudeten bis zum Alpenbogen. Ph. xanthocnemus ist hingegen auf die Pyrenäen beschränkt. Beide Taxa sind als typische Gebirgstiere auf die (hoch-)montane bis alpine Stufe beschränkt, so dass die Verbreitung nicht über das gesamte angegebene Gebiet verstanden werden kann, sondern als Teilfläche auf erhöhter Lage innerhalb dieses. Auf Grund dieser stärker eingeschränkten Verbreitung erhalten Populationen in den Pyrenäen für $P h$. xanthocnemus oder für $P h$. alpinus im Alpenbogen natürlich eine höhere Bedeutung.

Neben der oben gegebenen Revalidierung von Phyllobius alpinus Stierlin, 1859 muss nach Alonso-Zarazaga et al. (2017) Protapion ruficroides (Dieckmann, 1973) nun P. nigricrus (Desbrochers des Loges, 1895) heissen.

\section{Diskussion}

\section{Allgemein}

Eine Checkliste einer beliebigen Artengruppe ist stets kurz nach ihrem Erscheinen bereits wieder überholt; neue Funde werden gemacht, zusätzliche (auch alte Nachweise und Belege) werden entdeckt und die Nomenklatur ändert sich wie vorliegend aufgezeigt. Somit sind Nachträge zwecks einer ständigen Aktualisierung unverzichtbar, auch und besonders bei artenreichen Überfamilien wie den Curculionoidea. Die hier vorgestellten Funddaten betreffen folgende besonders erwähnenswerte Funde: Zwei Feuchtland-Arten (Drupenatus nasturtii und Neophytobius granatus) welche beide auf Grund fehlender und/oder stark im Rückgang begriffener Auenhabitate nur noch selten gefunden werden. D. nasturtii dürfte dabei von der Renaturierung im Gebiet beim Hechteloch (Kleinhochstettenau) profitiert haben, $N$. granatus wurde auf Überschwemmungsflächen im Naturschutzgebiet Kanderdelta als Beifang gefunden, eine schwer zugängliche Fläche. Zwei weitere Arten (Cossonus cylindricus und Gasterocercus depressirostris) leben xylobiont und die letztere Art, welche auch als Urwaldrelikt angesehen wird (Rheinheimer and Hassler 2010) - und von welcher neuerdings auch Belege aus dem Tessin bekannt geworden sind (teste L. Pollini) - scheint nun definitiv bei uns auch weiter 
verbreitet zu sein. Dieselbe Einschätzung von einem häufigeren Auftreten dürfte auch für Tychius cuprifer gelten, welcher im Gebiet beim Flughafen Mulhouse bei Basel (auf der französischen Seite) erst kürzlich im Mai 2019 in Dutzenden Exemplaren gefunden werden konnte. Bei Orchestes calceatus bleibt abzuwarten ob weitere Nachweise gelingen werden, die eher aus kühlen und feuchten Lebensräumen bekannte Art wird generell wenig gefunden. Curculio vicetinus ist ein Neobiont in Ausbreitung. Stenocarus ruficornis, welcher an Papaver-Arten lebt, dürfte davon profitieren, dass seine Wirtspflanze wieder in Getreideäckern toleriert wird. Die parthenogenetische und invasie Art Otiorhynchus indefinitus dürfte sich mit vorliegenden Funden definitiv bei uns etabliert haben und auch bereits weiter verbreitet sein.

Die letzten beiden vorgestellten Arten (Phrydiuchus topiarius und Donus intermedius) werden generell nur selten gefunden und sind bisher nur von kleinräumigen und isoliert liegenden Populationen bekannt geworden. Besonders im Hinblick auf die schwindende Biodiversität und das Insektensterben sind solche Fundmeldungen insbesondere von gefährdeten und wenig mobilen Arten von grosser Bedeutung und müssen publik werden.

\section{Nachträge zu historischen Literaturangaben und Änderungen der Nomenklatur}

Ein Beitrag zur Fauna des Kantons Glarus von Blösch (1875) wurde bisher übersehen, darin werden auch von Sackberg [bei Braunwald] und aus dem Klöntal acht Curculionoidea aufgelistet, die zum Teil bisher nicht in die Verbreitungsangaben der Checkliste Eingang fanden. Die faunistischen Beiträge von Rosset (1879) und Schacht (1879) zur Käferfauna des Wallis wurden bisher ebenfalls übersehen. Rosset (1879) führt 15 Curculionidae vom Simplon(pass) auf. Schacht (1879) gibt spannende Funde von 87 Curculionoidea überwiegend aus Sierre und Umgebung an, dabei einige Erstnachweise, zeitlich vor den bisherigen Meldungen von Favre (1890) wie der (noch immer unbestätigt gebliebene) walliser Fund von Protapion ruficrus (Germar, 1817) und die faunistisch interessanten Nachweise von Curculio elephas (Gyllenhal, 1836) und Trichosirocalus horridus (Panzer, 1801). Miot (1874) schliesslich führte einzelne Curculionidae, ohne besonders erwähnenswerte Nachweise, aus der Umgebung des Tarasper Bads (Graubünden) auf.

\section{Danksagung}

Hermann Blöchlinger (Erschmatt), Hansjörg Brägger (Amriswil), Stève Breitenmoser (Changins), Ruedi Bryner (Biel) und Werner Marggi (Allmendingen b. Thun) danke ich herzlich für die Möglichkeit der Überprüfung ihrer Belege und für die Verwendung der Funddaten. Ein herzliches Dankeschön gebührt den Reviewern für Ihre gründliche Durchsicht des Manuskripts.

\section{Literatur}

Alonso-Zarazaga MA, Barrios H, Borovec R, Bouchard P, Caldara R, Colonnelli E, Gültekin L, Hlaváč P, Korotyaev B, Lyal CHC, Machado A, Meregalli M, Pierotti H, Ren L, Sánchez-Ruiz M, Sforzi A, Silfverberg H, Skuhrovec J, Trýzna M, Velázquez de Castro AJ, Yunakov NN (2017) Cooperative Catalogue of Palaearctic Coleoptera Curculionoidea. Monografías electrónicas de la Sociedad Entomológica Aragonesa 8: 1-729.

Blösch C (1875) Deux excursions dans le canton de Glaris. Petites Nouvelles Entomologiques 1(126): 1-503.

Borer M, Germann C, Coray A, Luka H (2018) Kommentierte Artenliste der Käfer (Coleoptera) am GEO-Tag der Natur in den Merian Gärten 2017. Mitteilungen der Naturforschenden Gesellschaften beider Basel 18: 39-52.

Favre E (1890) Faune des Coléoptères du Valais et des Régions Limitrophes. Nouvelles Mémoires de la Société Helvétique des Sciences Naturelles 31, Imprimerie Zurcher \& Furrer, Zürich, 448 pp.

Germann C (2006) Beitrag zur Rüsselkäfer-Fauna der Schweiz - mit der Meldung von 17 weiteren Arten (Coleoptera, Curculionoidea). Mitteilungen der Schweizerischen Entomologischen Gesellschaft 79: 299-309.

Germann C (2010) Die Rüsselkäfer der Schweiz - Checkliste (Coleoptera, Curculionoidea) mit Verbreitungsangaben nach biogeografischen Regionen. Mitteilungen der Schweizerischen Entomologischen Gesellschaft 83: 41-118.

Germann C (2011) Supplement zur Checkliste der Rüsselkäfer der Schweiz (Coleoptera, Curculionoidea). Mitteilungen der Schweizerischen Entomologischen Gesellschaft 84: 155-169.

Germann C (2013) Erster Nachtrag zur Checkliste der Rüsselkäfer der Schweiz (Coleoptera, Curculionoidea). Mitteilungen der Schweizerischen Entomologischen Gesellschaft 86: 151-164.

Germann C (2014) Curculio vicetinus Cussigh, 1989 seit 2005 auch in der Schweiz (Coleoptera, Curculionidae). Mitteilungen der Schweizerischen Entomologischen Gesellschaft 87: 309-310.

Germann C (2017) Zweiter Nachtrag zur Rüsselkäfer-Fauna der Schweiz (Coleoptera, Curculionoidea). Alpine Entomology 1: 43-49 https://doi.org/10.3897/alpento.1.17788

Germann C, Chittaro Y (2019) Wiederfund und Bestätigung von Polydrusus sparsus Gyllenhal, 1834 für die Schweiz (Coleoptera, Curculionidae). Entomo Helvetica 12: 169-171.

Germann C, Graf R (2018) Massenfund und Beobachtungen zur Phänologie von Mecinus pascuorum (Gyllenhal, 1813) an der Alpennordflanke der Zentralschweiz (Coleoptera, Curculionidae). Entomologische Nachrichten und Berichte 62(3): 205-207.

Kraatz G (1874) Ueber Orchestes pubescens Steph. und semirufus Gylh. Mitteilungen der Schweizerischen Entomologischen Gesellschaft 4: 279-280.

Miot M (1874) Nouvelles. Petites Nouvelles Entomologiques 106: $1-423$.

Pesarini C (1981) Le specie paleartiche occidentali della tribù Phyllobiini (Coleoptera Curculionidae). Bollettino di Zoologia Agraria e di Bachicoltura (ser. n.) 15: 49-230.

Rheinheimer J, Hassler M (2010) Die Rüsselkäfer Baden-Württembergs. Verlag Regionalkultur, Heidelberg, 944 pp.

Rosset C (1879) Contributions à la faune entomologique du Valais. Insectes rares capturés sur le Simplon. Bulletin des Travaux de la Société murithienne du Valais 9: 36-38. 
Schacht [?] (1879) III Contribution à la faune des Coléoptères du Valais. Bulletin des Travaux de la Société murithienne du Valais 9: 43-54.

Stierlin G (1898) Fauna Coleopterorum Helvetica. Teil II. Bolli und Boecherer, Schaffhausen, $662 \mathrm{pp}$.

Stierlin G (1883) Zweiter Nachtrag zur Fauna coleopterorum helvetica. Denkschriften der schweizerischen Gesellschaft für die gesammten Naturwissenschaften 28(3): 60-81.
Stüben PE, Bayer C, Bahr F, Sprick P, Behne L (2015) Digital-Weevil-Determination der westpaläarktischen Curculionoidea: Transalpina: Phyllobiini. Snudebiller: Studies on taxonomy, biology and ecology of Curculionoidea 16 (240): 1-14. 